Research Paper

\title{
The Pro-angiogenesis Of Exosomes Derived From Umbilical Cord Blood Of Intrauterine Growth Restriction Pigs Was Repressed Associated With MiRNAs
}

Jia Luo ${ }^{1, \dagger}$, Yuan Fan 1,t, Linyuan Shen ${ }^{1}$, LiLi Niu1, Ye Zhao ${ }^{1}$, Dongmei Jiang1, Lin Zhu², An'an Jiang1, Qianzi Tang ${ }^{1}$, Jideng Ma1, Long Jin ${ }^{1}$, Jinyong Wang ${ }^{3}$, Xuewei Li $^{1}$, Shunhua Zhang ${ }^{\circledR}$, Li Zhu ${ }^{\circledR}$

1. College of Animal Science and Technology, Sichuan Agricultural University, Chengdu, Sichuan, China.

2. Mianyang Ming Xing Agricultural Science and Technology Development Co., LTD, Mianyang, Sichuan, China.

3. Chongqing Academy of Animal Science, Rongchang 402460, China

† These authors contributed equally to this work.

$\triangle$ Corresponding authors: zhangsh1919@163.com (SZ); zhuli7508@163.com (LZ)

(C) Ivyspring International Publisher. This is an open access article distributed under the terms of the Creative Commons Attribution (CC BY-NC) license (https://creativecommons.org/licenses/by-nc/4.0/). See http://ivyspring.com/terms for full terms and conditions.

Received: 2018.05.03; Accepted: 2018.07.10; Published: 2018.08.06

\begin{abstract}
Dysfunctional umbilical cord blood (UCB) is a key factor for the development of intrauterine growth restriction (IUGR) in utero. Poor degrees of angiogenesis were observed during IUGR development. Here, it was demonstrated that NV-EXO (normal piglet's Umbilical Veins derived exosomes) promoted angiogenesis within the subdued pro-angiogenesis context of IV-EXO (IUGR piglet's Umbilical Veins derived exosomes). Investigation of the miRNA transcriptome of umbilical cord vein and artery exosomes between IUGR and normal littermates showed significant differences between umbilical veins from normal (NV) and IUGR (IV) piglets. Similar patterns were observed in normal (NA) and IUGR (IA) umbilical arteries as well. Moreover, the miRNAs expession level was more stable in NV. Further analysis revealed that miRNAs related to angiogenesis exhibited aberrant expression in IUGR pigs. The miRNA expression patterns between IUGR and normal piglets showed great difference. Expression of miR-150 in the tissues and UCB exosomes of IUGR pigs was significantly decreased. Up-regulation of miR-150 was able to increase proliferation, migration and tube formation of Human umbilical vein endothelial cells (HUVECs), suggesting a pro-angiogenic role. Furthermore, the data demonstrated that $\mathrm{UCB}$ derived miRNAs participate in fetal epigenetic regulation during pregnancy, suggesting a novel possible explanation for abnormal embryologic vascular development and several congenital cardiovascular diseases.
\end{abstract}

Key words: IUGR; pig; umbilical cord blood; exosomes; angiogenesis; miRNA

\section{Introduction}

Intrauterine growth restriction (IUGR) is normally defined as the failure of a fetus to reach its genetically pre-determined growth potential, which represents one of the most common and complex problems in modern obstetrics[1]. Affecting approximately $5-10 \%$ of human neonates worldwide, and $15-20 \%$ of newborn piglets, IUGR is widely considered to be a major problem in human medicine and animal production [1, 2]. These fetuses with impaired fetal programming and growth restriction are at a risk for developing chronic diseases of adulthood, including various neurological disorders and cardiovascular diseases $[3,4]$.

The causes of IUGR are generally described under 3 main categories: maternal, fetal, and placental [5]. The umbilical cord is the physical link between the placenta and fetus. Structurally, the umbilical cord consists of 2 umbilical arteries (UA) and 1 umbilical 
vein (UV). It contributes to placenta-fetal communication, and plays an indispensable role in the intrauterine embryonic development. Studies have reported that intrauterine growth-restricted fetuses had a higher proportion of lean umbilical cords [6]. The umbilical cord changes, with respect to the physical dimensions and composition, can be considered reflective of changes in the fetal cardiovascular system [7]. Furthermore, angiogenesis occurs during numerous normal physiological conditions (e.g. embryogenesis, reproduction, and wound healing) as well as in many pathological processes $[8,9]$. Exosomes are membranous vesicles (30-100 $\mathrm{nm}$ in diameter) that are released by a variety of cell types into the extracellular space. Furthermore, exosomes are considered to be important mediators of intercellular communication for immune signaling, stress responses, and angiogenesis etc. Recent studies have suggested that exosomes play a significant role in cellular communications [10-13], disposal of metabolic waste, and the trans-placental transfer of nutrients to the developing fetus [14]. Ouyang et al. have suggested that placental-derived exosomes and their biological contents (RNAs and protein) contributed to maternal-fetal communication during pregnancy [15]. Moreover, exosomes deliver a significant proportion of circulating miRNAs [16]. These implied miRNAs in Umbilical cord blood (UCB) exosomes could be a new possible mechanism involved in the development of IUGR. However, no publications studied the role of miRNAs in UCB exosomes during in utero embryonic development.

Pigs (Sus scrofa) are emerging as an attractive biomedical model for the study of human diseases due to their similar anatomy, genetics, and physiological characteristics, compared to mice and other mammals $[17,18]$. Indeed, pig models offer the advantages of homogeneous feeding regime, and are absent many confounding factors typical for humans. In the modern swine industry, pigs have undergone strong genetic selection in relatively inbred commercial lines, which has led to remarkable phenotypic changes and genetic adaptation. Therefore, these breeding lines are a perfect model for IUGR studies [19]. In the present study, the Umbilical cord morphological and histological characteristics were analyzed, as well the pro-angiogenesis functions of UCB exosomes. Moreover, miRNA transcriptome profiles of UV and UA exosomes from IUGR and normal littermates were evaluated. The data suggested that the angiogenesis inducing capability of IUGR was poor. Although NV-EXO exhibited pro-angiogenesis properties, those of IV-EXO were markedly diminished. Aberrant expression of miRNAs derived from UCB was observed in IUGR piglets; miR-150 was down-regulated in exosomes derived from IUGR's UCB, and was capable of promoting angiogenesis. The present study contributes to an improved understanding of the influence of miRNAs contained within UCB exosomes on fetal epigenetic regulation during pregnancy, while exploring a novel possible mechanism for the formation of IUGR.

\section{Materials and Methods}

\subsection{Experimental samples}

Yorkshire piglets were obtained from the Mianyang Ming Xing Agriculture Science and Technology development Co., LTD, (Mianyang, Sichuan, China). All studies were performed in strict accordance with the Regulations for the Administration of Affairs Concerning Experimental Animals (Ministry of Science and Technology, China, revised in June 2004). All newborn Yorkshire piglets used for collecting umbilical cords and UCB were born naturally, had no genetic defects, and a clear genetic background. All the piglets were gave birth at full term (113 $\pm 2 d$ gestation). In this study, 3 litters were selected using the IUGR birth weight criterion (i.e. a birth weight of 1.5 SD below the mean birth weight of the litter)[20]. As a result, 6 newborn male piglets from the 3 litters (treating the lightest one from each litter as IUGR and the heaviest one as normal) were selected. Exosomes in UA and UV were utilized for RNA isolation, and were then used for library construction, q-PCR analysis, and in vitro experiments. Umbilical cord samples and other tissues were collected for immunohistochemistry and q-PCR analysis.

\subsection{Histological and immunohistochemistry analysis}

Tissues were harvested for the histological examination, washed thrice with phosphate-buffered saline (PBS) to remove blood, and were then fixed in $4 \%$ paraformaldehyde. Umbilical cord segments were embedded in paraffin blocks and stained with hematoxylin and eosin after fixation. A rabbit monoclonal antibody specific to CD34 was purchased from Abcam (USA). Immunohistochemical analysis of CD34 expressing cells was performed by the Google Biotechnology limited company (Chengdu, China). CD34+ light density were analyzed by Image-pro plus 6.0 (Media Cybernetics, Inc., Rockville, MD, USA)

\subsection{UCB Collection, exosome preparation and identification}

An anticoagulant solution of $15 \%$ EDTA was prepared, and 2 drops were combined in a $5 \mathrm{ml}$ syringe before collecting umbilical cord blood. At the 
time of birth, the piglets were placed under a heat lamp, UA and UV blood samples were collected from the umbilical cord. Samples were inverted several times to ensure inhibition of clotting by the EDTA solution. The blood was then transferred to $2 \mathrm{ml}$ centrifuge tubes ( $1 \mathrm{ml}$ per tube). Exosomes in the UCB were isolated as previously described by Théry et al. [21]. The umbilical cord blood exosomes were prepared as previously described by Palanisamy et al. [22]for atomic force microscopy (AFM) analysis. The exosome suspensions were diluted 1:500 in deionized water, and were then adsorbed to freshly cleaved mica sheets for $10 \mathrm{~min}$. Excess exosome suspension was carefully removed with absorbent filter paper, and the mica was further dried before analysis. Surface morphology was examined under an atomic force microscope (Asylum Research MFP-3D-Bio, Digital Instruments Inc., Santa Barbara, CA).

\subsection{RNA isolation and $q-P C R$}

Total exosomal RNA (including miRNAs) were extracted using the TRIzol LS reagent (Invitrogen) according to the manufacturer's instructions. Total RNA quality and concentration were estimated by gel electrophoresis and a spectrophotometry (Thermo, Waltham, MA, USA). Reverse transcriptions of microRNAs were performed using a commercial kit (TaKaRa, China), according to the manufacturer's instructions. Quantitative real-time PCR (qRT-PCR) of miRNAs were performed using a SYBR Premix Ex Taq kit (TaKaRa, China) on a Bio-RAD IQTM5 system (Bio-Rad, Hercules, CA, USA). The amplification conditions were as follows: initial denaturation at 95 ${ }^{\circ} \mathrm{C}$ for $30 \mathrm{~s}$, followed by 40 cycles of denaturation at 95 ${ }^{\circ} \mathrm{C}$ for $30 \mathrm{~s}$, annealing at $60^{\circ} \mathrm{C}$ for $40 \mathrm{~s}$, and extension at $72{ }^{\circ} \mathrm{C}$ for $30 \mathrm{~s}$. The forward primer of miRNAs was identical in sequence and length to the miRNA itself (i.e., the most abundant isomiR) based on our sequencing results. The expression levels of individual miRNAs were normalized to miRNA U6. Relative expression of miRNAs was calculated via the $2^{-\Delta \Delta C t}$ method.

\subsection{Small RNA Library Construction and Sequence Analysis}

Samples from either the 3 normal or 3 IUGR piglets were pooled respectively and used for the construction of small RNA libraries. Total RNA was extracted using Trizol LS reagent (Invitrogen, CA, USA) following the manufacturer's procedure. The total RNA quantity and purity were analysis of Bioanalyzer 2100 and RNA 6000 Nano LabChip Kit (Agilent, CA, USA) with RIN number $>7.0$. Approximately $1 \mu \mathrm{g}$ of total RNA was used to prepare the small RNA libraries according to the protocol of the TruSeq Small RNA Sample Prep Kits (Illumina). Single-end sequencing ( $36 \mathrm{bp}$ ) was performed on an Illumina Hiseq 2500 at the LC-BIO (Hangzhou, China), following the recommended protocol. The small RNA sequencing data were uploaded to NCBI's Gene Expression Omnibus (GEO), and can be found under Accession Number GSE87111.

The procedures used here for data processing have been described previously [23]. The raw reads were subjected to the Illumina pipeline filter (Solexa 0.3 ), and the dataset was further processed using ACGT101-miR (LC Sciences, Houston, TX) in order to remove adapter dimers, poor quality sequences, low complexity, common RNA families (rRNA, tRNA, snRNA, and snoRNA), and repeats. Then the high-quality reads were mapped to the pig genome (Sscrofa10.2) using NCBI Local BLAST following three steps in order: (1) map the high-quality reads to the precursor miRNAs of pig and 24 other mammals in miRbase 21.0; (2) map the mapped high-quality reads to pig genome (Sscrofa10.2) to obtain their genomic locations and annotations using NCBI Local BLAST; (3) Unique sequences with lengths of 18-26 nt were subsequently mapped to species-specific precursors in miRBase 21.0 through a BLAST search to identify known miRNAs and novel $3 p$ - and $5 p$-derived miRNAs. Sequences containing varied lengths at both the $3^{\prime}$ - and $5^{\prime}$-ends as well as a single mismatch within the sequence were retained in the alignment. Unique sequences mapping to species-specific mature miRNAs in hairpin arms were identified as known miRNAs.

\section{6. miRNA Target Gene Prediction and Pathway Analysis}

To predict target genes for the differentially expressed miRNAs, 2 bioinformatic algorithms (TargetScan 7.1 and miRanda 3.3a) were used to identify miRNA binding sites. The data predicted by both algorithms were combined, and overlaps were calculated. The GO terms and KEGG pathways of the differentially expressed miRNA targets were annotated.

\subsection{Cell Culture and miRNA Transfection}

Human umbilical vein endothelial cells (HUVECs) were kindly provided by the Medical College of Suzhou University. Cells were maintained at $37{ }^{\circ} \mathrm{C}, 5 \% \mathrm{CO}_{2}$ in DMEM (Hyclone, USA) supplemented with 10\% FBS (GIBCO, USA). The medium was changed every 2 days, and cells were passaged at $90 \%$ confluence. Cells were lifted from the culture vessel using $0.25 \%$ trypsin (Solarbio, China). Transfection of miRNAs into HUVECs was achieved using Hiperfect (QIAGEN, Germany), according the 
manufacturer's recommendation. Mimics of miRNAs or negative controls were purchased from RiboBio (Guangzhou, China). Total RNA was isolated $24 \mathrm{~h}$ after transfection.

\subsection{Cell proliferation assay}

HUVECs were seeded in 96-well plates and maintained in growth medium. The cells were then transfected with exosomes or miR-150 mimics, inhibitors, or negative control (NC). Cell proliferation was assessed using a Cell Counting kit 8 (DOJINDO, Japan) according to manufacturer's protocol. For Edu analysis, HUVECs were treated with $10 \mu \mathrm{m}$ Edu for $24 \mathrm{~h}$ following transfection and incubated for $14 \mathrm{~h}$. Staining for Edu was performed according to the manufacturer's protocol. Images were captured using an Olympus IX53 microscope (Olympus, Tokyo, Japan).

\subsection{Scratch Assay and Tube Formation Assay}

The cells were transfected and grown to near confluence in 12-well plates. Next, the HUVEC monolayers were scratched with a $200-\mu l$ pipette tip. Non-adherent cells in the culture supernatants and cell debris were removed by 3 washes with PBS. After incubating the HUVECs in DMEM containing $100 \mu \mathrm{g} / \mathrm{ml}$ exosomes or PBS, or miR-150 or negative control, the cells were allowed to recover for $24 \mathrm{~h}$. Photos were taken at the same fields of view at $0 \mathrm{~h}$ and $24 \mathrm{~h}$ after scratching the monolayer. "Wound healing" was assessed by analyzing images in ImageJ Pro 1.51. Matrigel (CORNING, USA) was added to each well of the 96-well plate, and allowed to polymerize at $37{ }^{\circ} \mathrm{C}$ for $30 \mathrm{~min}$. The HUVECs were then treated with exosomes $(100 \mu \mathrm{g} / \mathrm{ml})$ or PBS, or miR-150 mimics or negative control for $24 \mathrm{~h}$. The de novo formed capillary-like structures were imaged using a Nikon TE2000 microscope (Nikon, Japan).

\subsection{Statistical analysis}

Data are presented as means \pm SEM. Differences between groups were analyzed using a Student's two-tailed $t$-test for independent samples.

\section{Results and Discussion}

\subsection{Angiogenesis of IUGR was repressed}

Aberrant vascular formation is believed to be a possible contributing factor to the development of IUGR [24]. Furthermore, it has been reported that IUGR fetuses had a higher proportion of lean umbilical cords [6]. To determine the differences exist in umbilical cords of IUGR and normal piglets, the lightest and the heaviest piglets from 3 litters (the average litter weights were $2.02 \pm 0.42 \mathrm{~kg}, 1.87 \pm 0.29$ $\mathrm{kg}$, and $1.94 \pm 0.33 \mathrm{~kg}$, respectively) were selected based on an individual with a birth weight of $1.5 \mathrm{SD}$ below the mean birth weight of its litter [25]. The average weights of IUGR and normal piglets were $0.84 \pm 0.18 \mathrm{~kg}$ and $1.90 \pm 0.32 \mathrm{~kg}$, respectively (Fig. 1A). The developmental progress and survival rates depended primarily on the functional integrity of the maternal-placental-fetal circulation. In fact, placental development precedes fetal development, suggesting that placental dysfunction is a contributing factor to the development of IUGR [26, 27]. One important stage of placental development is the formation of adequate vasculature during gestation to satisfy the demands of the growing fetus [28]. The vascular changes occurring in the placental bed and in the microcirculation of the placenta are reflective of the morphometric changes observed in umbilical vessels. The UC, as a physical link between placenta and fetus, are extremely long and muscular. Analysis of several UC parameters, specifically diameter (UV-D) and cross sectional area (UV-CSA), was significantly lower in IUGR pigs than in controls. A similar observation was observed in the UA and UV as well (Fig. 1B, Table 1). This finding was consistent with those of previously published studies in humans [7]. The umbilical cord serves as a conduit for blood flow to the developing fetus, and is considered to be an extension of the fetal cardiovascular system. Changes in the physical dimensions and tissue composition of the UC can be reasonably inferred to be reflective of changes in fetal tissue vasculature [7]. Furthermore, immunohistochemical analysis of CD34 expression in tissues suggested that angiogenesis in IUGR pigs was reduced compared to that of normal piglets in the brain, liver, cerebellum and heart (Fig. 1C, D). In addition, it was observed that the expression level of VEGF was lower in tissues from IUGR piglets compared to normal piglets (Fig. 1E). These data indicate that angiogenesis was repressed in IUGR pigs.

Table 1. The various morphometric parameters measured on the cord

\begin{tabular}{|c|c|c|c|}
\hline Umbilical cord parameters & Normal & IUGR & $\mathrm{P}$ \\
\hline UC-D (mm) & $6.2(4.27,7.59)$ & $4.12(2.75,5.86)$ & $<0.001$ \\
\hline UC-CSA $\left(\mathrm{mm}^{2}\right)$ & $32.57(26.2,45.89)$ & $27.44(19.37,36.55)$ & $<0.001$ \\
\hline WJA $\left(\mathrm{mm}^{2}\right)$ & $22.77(16.35,31.09)$ & $17.35(11.26,24.95)$ & $<0.001$ \\
\hline UV-D (mm) & $2.06(1.73,2.42)$ & $1.79(1.54,2.46)$ & $<0.001$ \\
\hline UV-CSA $\left(\mathrm{mm}^{2}\right)$ & $3.50(3.06,5.86)$ & $3.19(2.08,5.14)$ & $<0.001$ \\
\hline UV-LCSA $\left(\mathrm{mm}^{2}\right)$ & $1.53(0.69,2.26)$ & $1.45(0.43,2.08)$ & $<0.05$ \\
\hline UA-D (mm) & $0.99(0.54,1.28)$ & $0.67(0.58,1.20)$ & $<0.001$ \\
\hline UA-CSA $\left(\mathrm{mm}^{2}\right)$ & $2.41(1.64,3.38)$ & $1.96(1.35,2.41)$ & $<0.01$ \\
\hline UA-LCSA $\left(\mathrm{mm}^{2}\right)$ & $0.34(0.16,0.52)$ & $0.32(0.21,0.68)$ & $>0.05$ \\
\hline \multicolumn{4}{|c|}{$\begin{array}{l}\text { Note: Umbilical cord (UC); Umbilical cord diameter (UC-D); Umbilical cord total } \\
\text { cross-sectional area (UC-CSA); Umbilical vein (UV); Umbilical vein diameter } \\
\text { (UV-D); Umbilical vein cross-sectional area (UV-CSA); Umbilical vein luminal } \\
\text { cross-sectional area (UV-LCSA); Umbilical artery (UA); Umbilical artery diameter } \\
\text { (UA-D); Umbilical artery cross-sectional area (UA-CSA); Umbilical artery luminal } \\
\text { cross-sectional area (UA-LCSA). The numbers in the parentheses means the } \\
\text { minimum and maximum values of this term. }\end{array}$} \\
\hline
\end{tabular}


A

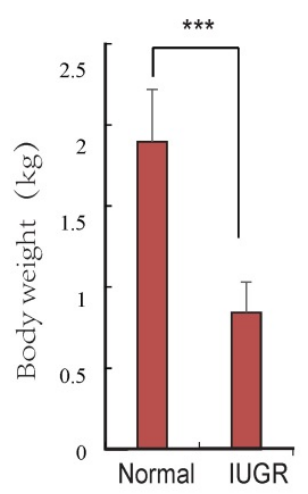

C

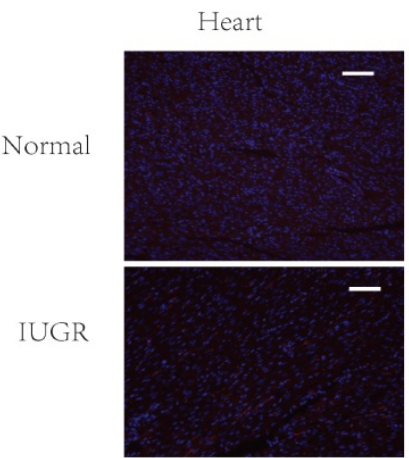

D

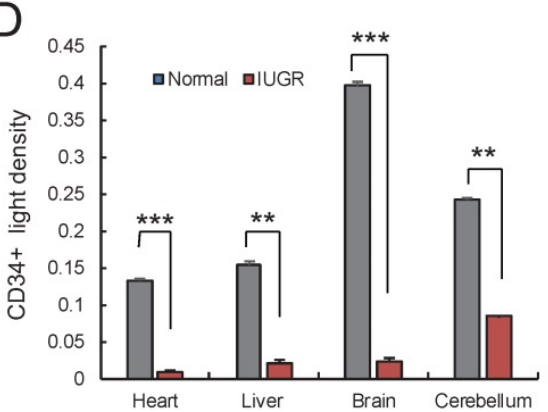

B

Liver
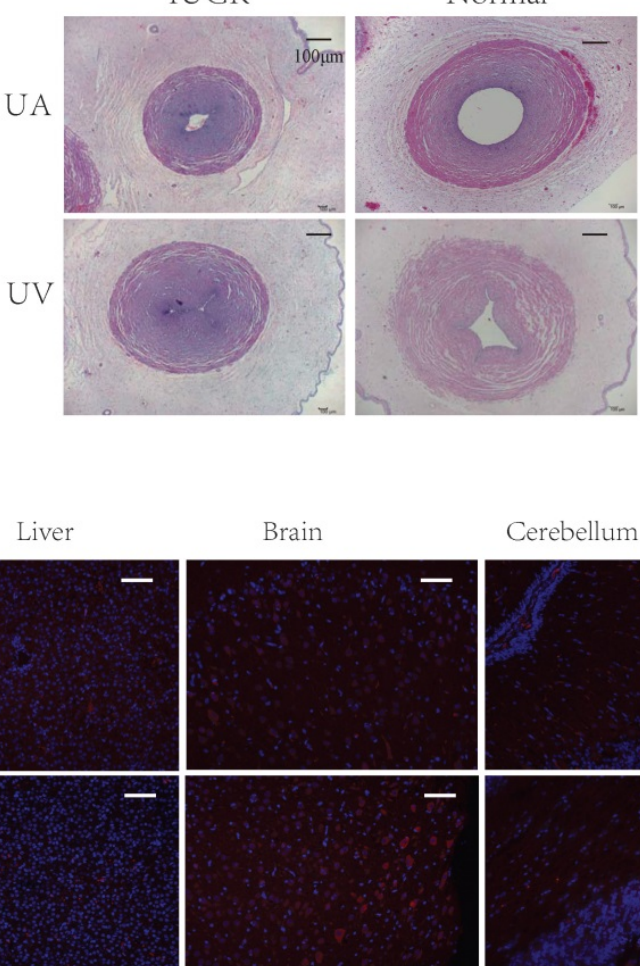

Cerebellum

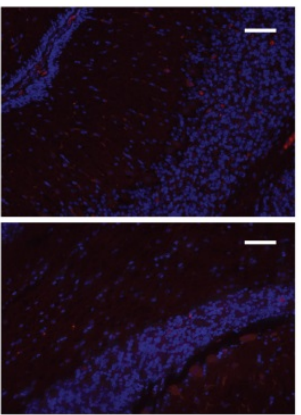

E

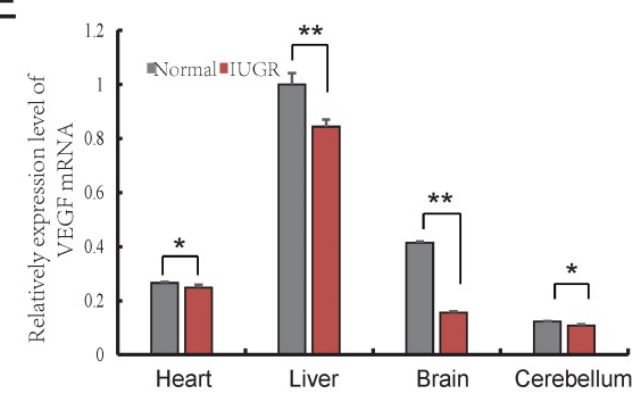

Fig. 1. Angiogenesis of IUGR pig was repressed. (A) Body weight of the IUGR and normal piglets; (B) The Characteristics of Umbilical Cord, $40 \times$, scale=100 $\mu m$. umbilical artery of IUGR (IA); umbilical artery of normal (NA); umbilical vein of IUGR (IV); umbilical vein of normal (NV); (C) CD34 immunohistochemistry of both normal and IUGR piglet's brain, liver, cerebellum and heart. Red represents CD34 protein, cell nuclei were stained with DAPI (blue). (D) CD34 positive light density, Red represents CD34 protein. (F) VEGF mRNA relative expression level in both normal and IUGR piglet's tissues. * or **represent the significance at the level of 0.05 or 0.01 , n.s represent non-significant, ****represent the significance at the level of 0.001 .

\subsection{The pro-angiogenesis role of IV-EXO was repressed}

Umbilical cord blood plays an indispensable role in placenta-fetal communication. Torry et al. reported an association between IUGR and aberrant angiogenesis during fetal development [29]. Several other reports have suggested that exosomes in the UCB could be a new factor contributing to abnormal vascular development during IUGR. In the present study, exosomes isolated from UCB were investigated using atomic force microscopy (AFM) at nanometer scale resolution. Porcine UCB contains a substantial amount of exosomes, measuring approximately 120 $\mathrm{nm} \times 20 \mathrm{~nm}$, presenting as flattened, donut-like structures similar to exosomes observed in human saliva and breast milk (Fig. 2A, B) [22, 30]. To investigate whether UCB exosomes participated in angiogenesis, HUVECs were selected for the analysis. These cells are a valuable in vitro model of angiogenesis due to their ability to form capillary-like structures, referred to as tubes, in response to appropriate stimuli [31]. Since the umbilical vein is the main supply to the fetus, and with increasing evidence showing hypoplasia of umbilical vein in IUGR, the roles of exosomes derived from UV were explored to evaluate its pro-angiogenesis activities. The results indicated that NV-EXO enhanced cell growth by increasing the neonatal proliferation of HUVECs. However, IV-EXO decreased this proliferation (Fig. 2C, D). The scratch assay was conducted using HUVECs co-cultured with EXO-free, NV-EXO, IV-EXO to determine their influence of 
motility of the cells. The data indicated that NV-EXO significantly promoted HUVEC migration. In contrast, the migration of HUVECs was significantly reduced in the presence of IV-EXO (Fig. 2E, F). The pro-angiogenesis genes VEGF and Notch1 were observed to be up-regulated when cells were cultured in the presence of NV-EXO, however IV-EXO resulted in down-regulation of VEGF and Notch1 gene expression. However, the anti-angiogenesis gene TSP1 were observed to be down-regulated when cells were cultured in the presence of NV-EXO (Fig. 2G). In addition, co-culture with NV-EXO resulted in a significant increase in tube formation, whereas
IV-EXO decreased tube formation (Fig. 2H, I). These data indicated that NV-EXO was pro-angiogenesis, but IV-EXO was not. Previous studies have discovered human umbilical cord-derived MSCs (huMSCs) have therapeutic effects in the alleviation of acute and chronic kidney injury. This process is primarily mediated by exosome means [32, 33]. However, no reports have been published that focus directly on the function of UCB-derived exosomes. The data obtained in the present study demonstrated the potential for future therapeutic use of NV-EXO in humans.
A

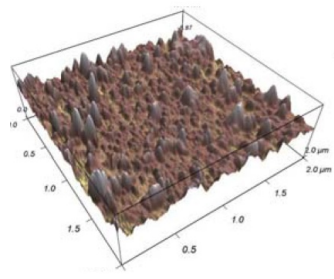

B

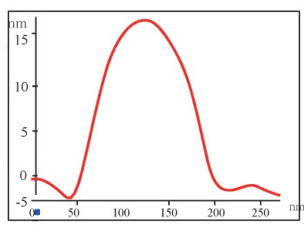

C EXO-free

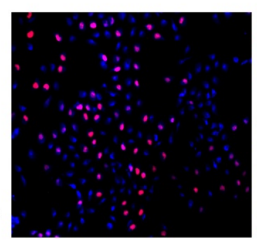

NV-EXO

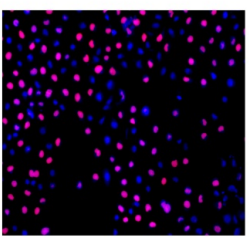

IV-EXO

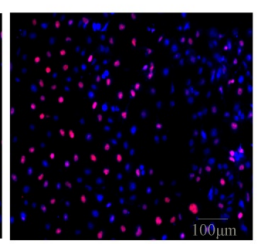

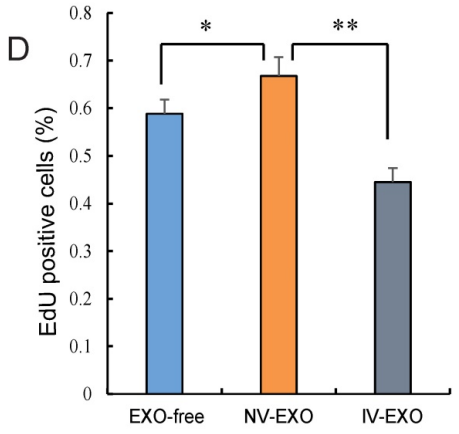

F

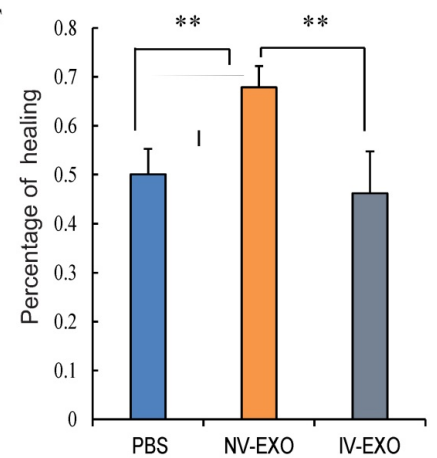

$\mathrm{H}$

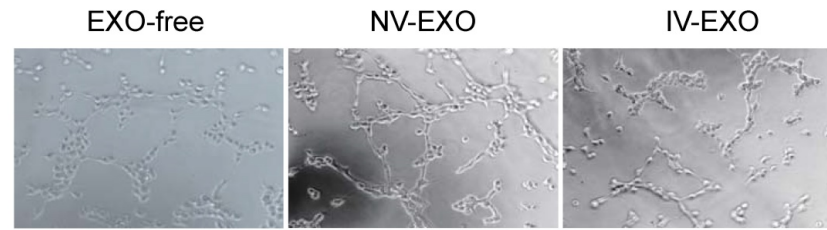

E EXO-free

G
NV-EXO

IV-EXO
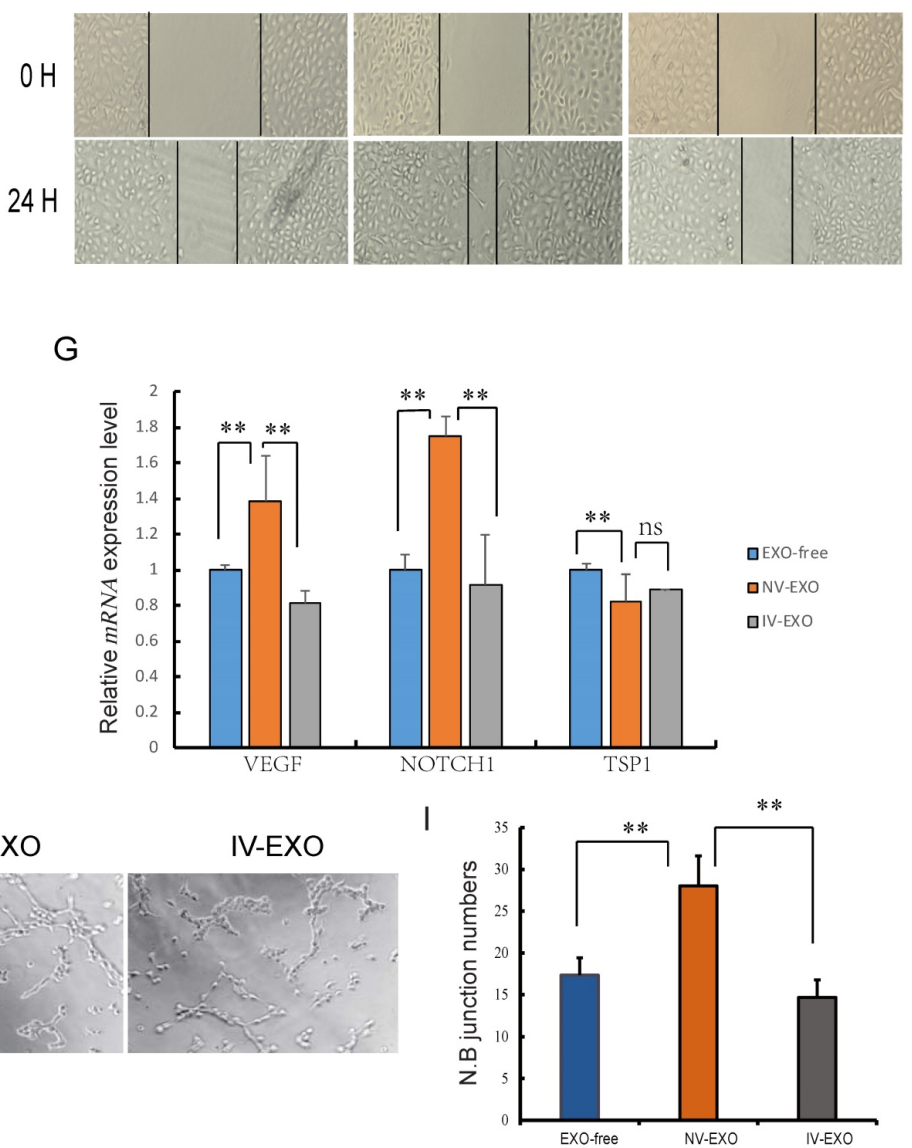

Fig. 2. The pro-angiogenesis role of IV-EXO was repressed. (A) The 3D morphology of isolated porcine umbilical cord blood exosomes observed in AFM; (B) The line profile of AFM image width for Umbilical Cord blood exosomes. X-and Y-axes are the width and height; (C) Cell viability was measured following exosomes or PBS treatment for 24h, using the Edu analysis; (D) Quantification of cell proliferation; (E) Evaluation of cell migration in vitro using a scratch assay in HUVECs following exosomes or PBS treatment for 24h ( $n=3)$; (F) Percentage of cell healing; (G) The expression level of VEGF and Notchl mRNA after treating exosomes or PBS; (H) Evaluation of angiogenesis in vitro using a matrigel assay in HUVECs following exosomes or PBS treatment $(n=3)$; (I) junction numbers of blood vessels. * or **represent the significance at the level of 0.05 or 0.01 , n.s represent non-significant. , $* * *$ represent the significance at the level of 0.001 . 


\subsection{UCB-EXO carrying angiogenesis-related miRNAs}

Angiogenesis is crucial for a wide variety of physiological and pathological processes. There has been a recent increase in evidence suggesting that miRNAs are important regulators of angiogenesis. Several miRNAs have been identified derived from exosomes, stimulating angiogenesis as well as facilitating metastatic development [34]. Thus, a comprehensive analysis of the miRNAs in UCB exosomes would facilitate a better understanding of the molecular mechanisms of IUGR development, as well as the therapeutic potential of UCB-EXO. To characterize the miRNA transcriptome of IUGR piglets in UCB exosomes, a deep sequencing approach was utilized [35]. As a result, 12.74 million (M), 13.29 $\mathrm{M}, 11.42 \mathrm{M}$, and $13.00 \mathrm{M}$ raw reads were obtained from NV, IV, NA, and IA exosomes, respectively (Table S1). The distribution of sequence lengths revealed that the majority of reads ranged from 21 to $24 \mathrm{nt}$ in length (accounting for $80 \%$ of the total reads) (Figure.S1). A total of 554 pre-miRNAs and 636 mature miRNAs were identified (Table 2). The vast majority $(52.71 \%)$ of all mapable sequences were identified as known precursor miRNAs (pre-miRNA), as determined by miRBase21.0. Moreover, the sequencing data of small RNAs were significantly and positively correlated with the observed qRT-PCR data, highlighting the reliability of the small RNA-sequencing approach (Fig. S2).

Table 2. Statistics of pre-miRNA and mature miRNA

\begin{tabular}{llllll}
\hline Classified & NV & IV & NA & IA & Total \\
\cline { 2 - 6 } & $\begin{array}{l}\text { miRNAs / } \\
\text { pre-miRNAs }\end{array}$ & $\begin{array}{l}\text { miRNAs } ~ \\
\text { pre-miRNAs }\end{array}$ & $\begin{array}{l}\text { miRNAs / } \\
\text { pre-miRNAs }\end{array}$ & $\begin{array}{l}\text { miRNAs / } \\
\text { pre-miRNAs }\end{array}$ & $\begin{array}{l}\text { miRNAs } / \\
\text { pre-miRNAs }\end{array}$ \\
\hline $\begin{array}{l}\text { Known } \\
\text { miRNAs } \\
\text { conservativ } \\
\text { e miRNAs }\end{array}$ & $248 / 224$ & $172 / 172$ & $178 / 172$ & $147 / 158$ & $292 / 260$ \\
$\begin{array}{l}\text { novel } \\
\text { miRNAs }\end{array}$ & $44 / 45$ & $28 / 28$ & $51 / 51$ & $38 / 38$ & $87 / 84$ \\
Total & $452 / 414$ & $288 / 452$ & $374 / 340$ & $273 / 278$ & $636 / 554$ \\
\hline
\end{tabular}

There are 2 pathways connecting the fetus and placenta, the UV and UA. In total, 116 and 226 miRNAs were observed to be differentially expressed (DE) between NV and IV, NA and IA $(P<0.01)$, whereas 74 miRNAs were co-expressed in both UV and UA $(P<0.01)$ (Fig. 3A). In addition, the top 10 unique miRNAs were observed in high abundance, accounting for $60 \%-70 \%$ of all total unique miRNA reads (Fig. 3B). Furthermore, these miRNAs are believed to function in important regulatory roles for the homeostasis of blood (e.g., erythropoiesis and angiogenesis). Interestingly, the expression profile of the most abundant (top 10) and unique miRNAs revealed striking similarities between NV and IV (Fig.
3B), which is in agreement with the miRNA cluster analyses conducted here (Fig. 3C). As vascular formation is important for fetal growth and development, it was observed that miRNAs promoting angiogenesis were down-regulated in IUGR. However, anti-angiogenesis miRNAs were up-regulated in the same animals. For example, miR-130a is believed to down-regulate the antiangiogenic homeobox genes GAX and HOXA5 to accelerate angiogenesis [36]. In the present study, expression of miR-130a was significantly lower in IUGR piglets than in normal animals. Furthermore, miR-126 plays an important role in the maintenance of vascular integrity, angiogenesis, and wound repair [37], and was found to be down-regulated in IUGR animals. GO and KEGG analyses indicated that the target genes of DE miRNAs were mainly associated with embryonic development, angiogenesis and blood vessel development (Fig. 3D). Previously published studies have reported that angiogenesis, as well as uterine and placental blood flow are crucial for mammals to maintain proper placental and fetal circulation, thus affecting embryonic growth [38]. Blood flow between uterus and fetus is the only route of substance exchange from the mother to fetus and from the fetus back to the mother. This hypothesis is consistent with our observations of altered vascular development and the growth rate of the embryo during embryonic development $[39,40]$. Furthermore, it was observed that miRNAs expressed in umbilical vein blood exosomes played a more central role in intrauterine development, whereas the UCB-EXO miRNAs were predominantly related to angiogenesis. Restrained angiogenesis may be an important factor leading to the development of IUGR.

\subsection{The NV-EXO promoted angiogenesis is partially mediated by the transfer of miR-150}

Intensive studies have provided evidence that exosomes released from many types of cell can transfer miRNAs to neighboring or distant cells. These exogenous miRNAs are able to function similarly to endogenous miRNAs with respect to the regulation of target gene expression and recipient cell function [41]. Because of their ability to form capillary-like structures called tubes in response to appropriate stimuli, HUVECs are a valuable in vitro model for the study of angiogenesis [31]. It was therefore hypothesized that UCB-EXO may affect HUVEC cell growth by the delivery of exogenous miRNAs. Based on sequencing results, miRNAs in UV were relatively stable, so the UV-derived exosomes were selected for the subsequent study. The expression of miR-150 has the greatest difference between normal and IUGR piglets both in umbilical 
arteries and umbilical veins exosomes. In particular, a strong enrichment of miR-150 in NV-EXO was observed relative to IV-EXO (Fig. 4A, B). It was also observed that IUGR animals had lower expression of miR-150 in tissues (Fig. 4C). Based on these observations, we sought to determine whether the high angiogenesis ability of NV-EXO is caused by the transfer of exogenous miR-150. To determine these effects, the HUVEC model was utilized in an EdU assay. As is presented in Fig. $4 \mathrm{D}$ and E, the HUVEC's neonatal cell proliferation ability was significantly enhanced after the introduction of miR-150. As the proliferation and migration of HUVECs are the key processes involved in angiogenesis, we also assessed whether miR-150 mediated proliferation and migration by CCK8 and scratch test. Following transfection of HUVECs with mimics of miR-150, the expression level of miR-150 increased significantly (Fig. 4F). Next, a cell viability assay was performed, and demonstrated a significant decrease in HUVEC viability (Fig. 4G). The migration ability of the

A
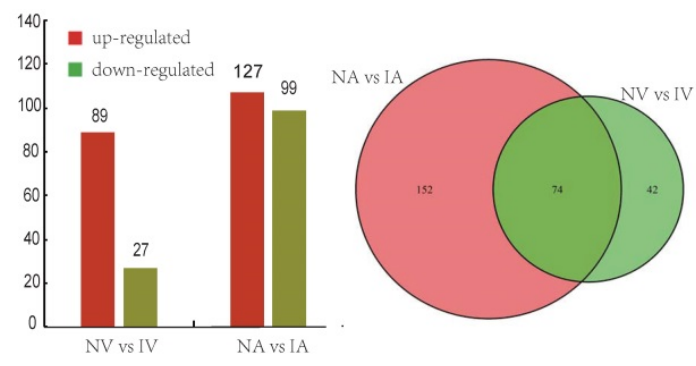

HUVECs was tested using a scratch assay. Following miR-150 mimic transfection, the percentage of cell monolayer re-growth was significantly improved (Fig. 4H, I). Analysis of VEGF and Notch1 expression by qRT-PCR confirmed that their expression levels increased in response to the up-regulation of miR-150, whereas the expression of ZEB1 was decreased (Fig. 4J). The tube formation assay showed HUVECs with increased miR-150 expression forms more novel cellular junctions compared to the NC (Fig. 4K, L). These results indicated that miR-150 in UCB-EXO may play a major role in angiogenesis in utero. In fact, the therapeutic use of UCB-EXO delivered miRNAs has long been thought be advantageous over traditional treatments [42]. In the present study, UCB-EXO was observed to participate in pro-angiogenesis functions, with miR-150 being a key regulator of angiogenesis in utero. These observations have shed light on a possible novel approach for the treatment of disease processes involving in altered angiogenesis.

B

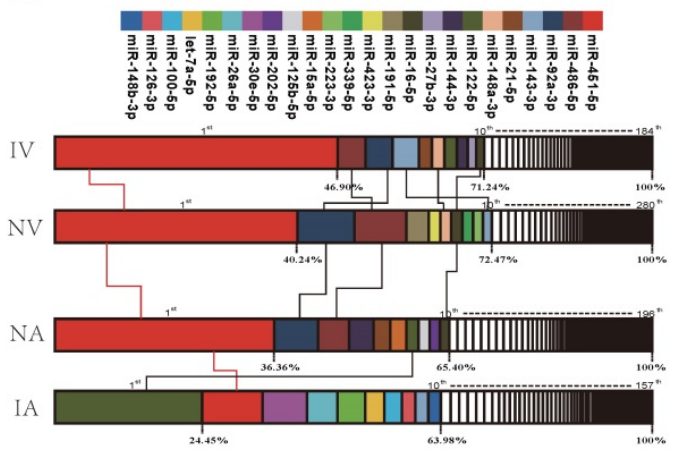

Fig. 3. UCB-EXO carrying angiogenesis-related miRNAs. (A) Differentially expressed (DE) miRNAs between NV (umbilical vein of normal) and IV (umbilical vein of IUGR), NA (umbilical artery of normal) and IA (umbilical artery of IUGR); Venn diagram of miRNAs expressed in NV, IV, NA and IA; (B) Analysis of miRNAs universally abundant across umbilical cord blood exosomes; (C) Cluster analysis of all expressed miRNAs. (D) GO and KEGG analysis of miRNAs differentially expressed (DE) between IUGR and normal littermates. Note: Red indicated the high expression of miRNA; Green indicated the low expression of miRNA. 

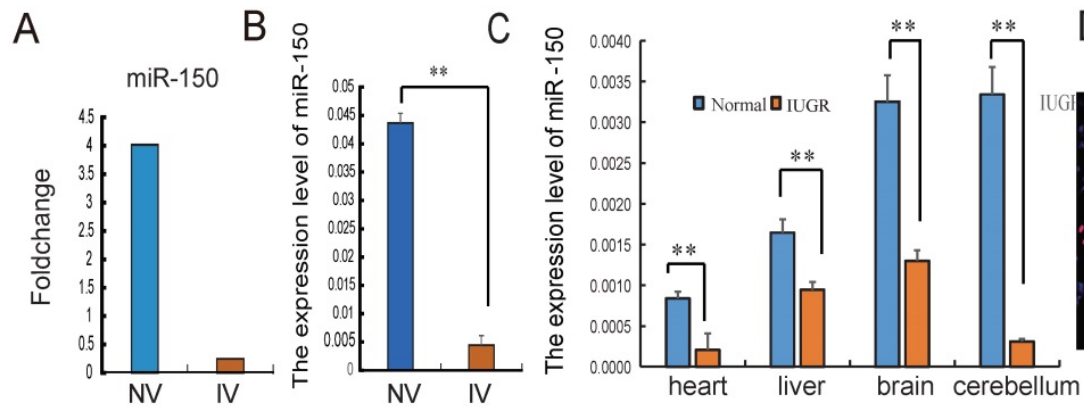

D

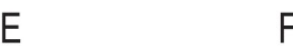

F G
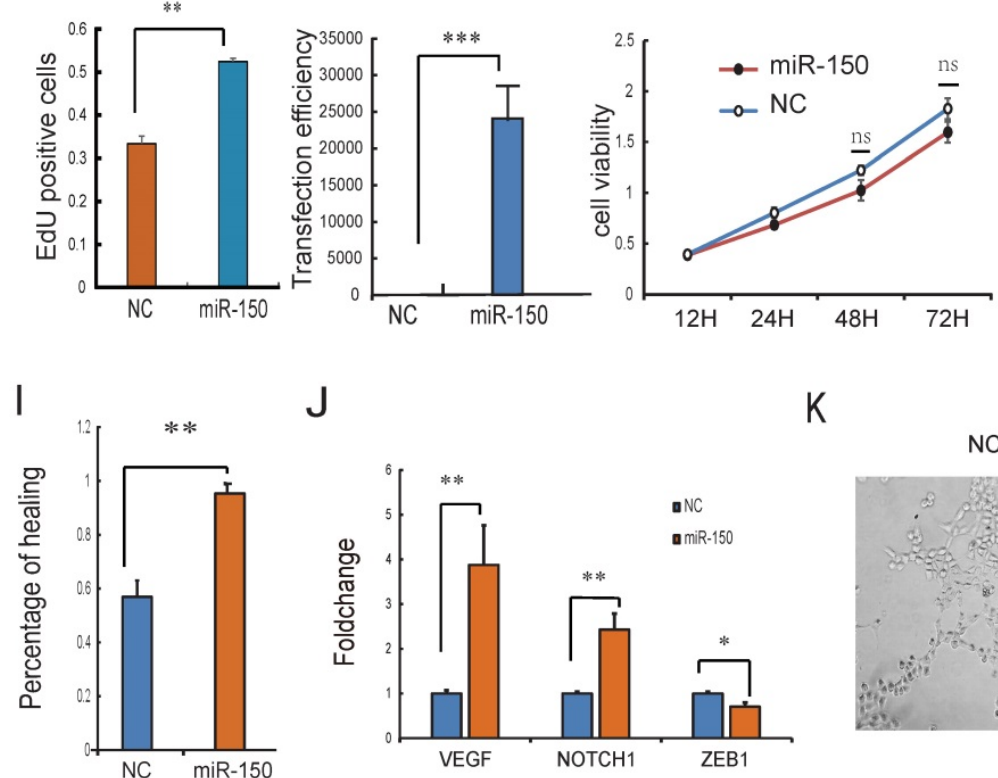

J

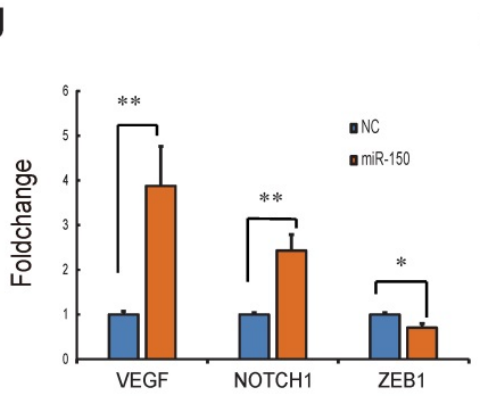

K

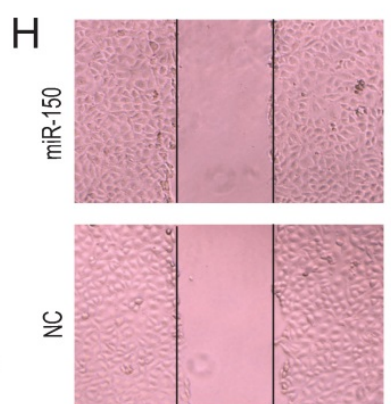

NC

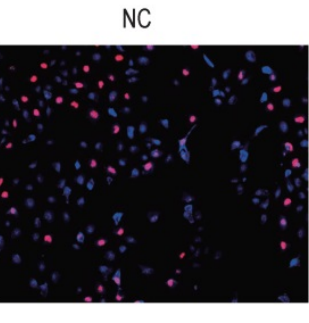

$\mathrm{OH}$

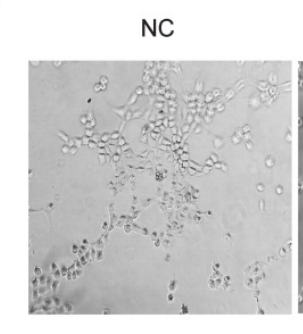

miR-150

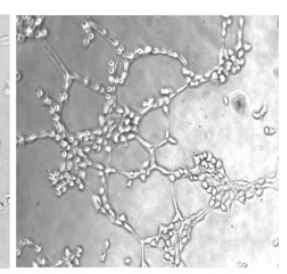

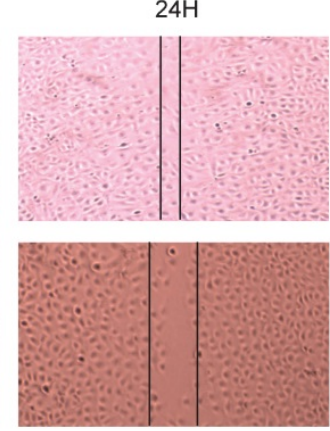

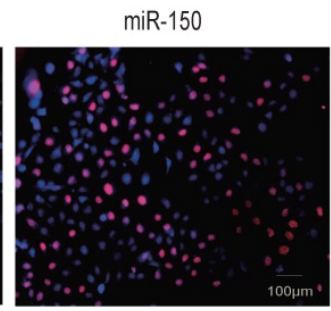

$24 \mathrm{H}$

$\mathrm{L}$

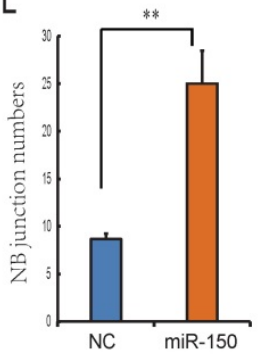

Fig. 4. The NV-EXO promoted HUVEC cell growth is partially mediated by transferring miR-150. (A) Foldchange of the miR-150 expression between NV and IV; (B) The expression level of miR-150 in NV and IV by q-PCR; (C) the expression level of miR-150 in IUGR and normal pig tissues; (D) (E) Cell viability after miR-150 mimics treatment using Edu test; (F) Fold change of miR-150 in HUVECs after treating miR-150 mimic, Negative control was treated with a scrambled miR mimic control; (G) Cell viability was measured following miR-150 mimics or a scrambled miR mimic control treatment, using the cell count kit $8(n=6)$; $(H)(I)$ Evaluation of cell migration in vitro using a scratch assay in HUVECs following miR-150 mimics or a scrambled miR mimic control treatment for 24h ( $n=3$ ); (J) The expression level of VEGF, Notch1 and ZEB1 mRNA after treating miR-150 mimics or a scrambled miR mimic control; $(\mathrm{K})$ (L) Evaluation of angiogenesis in vitro using a matrigel assay in HUVECs following miR-150 mimics or a scrambled miR mimic control treatment $(n=3)$, * or ** represent the significance at the level of 0.05 or 0.01 , n.s represent non-significant.

\section{Conclusion}

Taken together, the results of the present study suggest that angiogenesis is repressed during IUGR intrauterine embryonic development. The UCB-derived exosomes took part in the regulation of endothelial cell functions, possessed pro- angiogenic characteristics, which was at least partially attributable to the miRNAs contained within. Although intrauterine angiogenesis is regulated by various factors, exosomal miRNAs may play significant regulation roles in this important process. Our study developed a novel explanation for the development of IUGR, as well as a new direction through which to study epigenetic phenomena and congenital diseases.

\section{Abbreviations}

IUGR: intrauterine growth restriction

UC: umbilical cord
UCB: umbilical cord blood

UC-D: umbilical cord diameter

UC-CSA: umbilical cord total cross-sectional area

UV-D: umbilical vein diameter

UV-CSA: umbilical vein cross-sectional area

UV-LCSA: umbilical vein luminal cross-sectional area

UA-D: umbilical artery diameter

UA-CSA: umbilical artery cross-sectional area

UA-LCSA: Umbilical artery luminal cross-sectional area

UV-D: umbilical vein diameter

UV-CSA: umbilical vein cross sectional area

UA: umbilical cord artery

UV: umbilical cord vein

NA: umbilical artery of normal

IA: umbilical artery of IUGR

IV: umbilical veins of IUGR

$\mathrm{NV}$ : umbilical veins of normal 


\section{Supplementary Material}

Supplementary figures and table.

http://www.ijbs.com/v14p1426s1.pdf

\section{Acknowledgements}

This work was supported by grants from the National Natural Science Foundation of China (31530073), the Chinese National Sci \& Tech Support Program (No. 2015BAD03B01-11), the Sichuan Sci \& Tech Support Program (No. 16ZC2838; No. 2016NZ0089), the earmarked fund for China Agriculture Research System (No. CARS-36-05B).

\section{Authorship}

Conceived and designed the experiments: Jia Luo, Yuan Fan, Shunhua Zhang, Li Zhu. Performed the experiments: Jia Luo, Linyuan Shen, LiLi Niu, Ye Zhao, Dongmei Jiang. Analyzed the data: Jia Luo, Lin Zhu, Jinyong Wang, Dengji Ma, Xuewei Li. Contributed reagents/materials/analysis tools: Jia Luo, Lin Zhu, An'an Jiang, Qianzi Tang, Long Jin, Li Zhu. Wrote the paper: Jia Luo, Linyuan Shen, Li Zhu. All authors read and approved the final manuscript.

\section{Competing Interests}

The authors have declared that no competing interest exists.

\section{References}

1. Wu G, Bazer F, Wallace J, Spencer T. Board-invited review: intrauterine growth retardation: implications for the animal sciences. Journal of animal science. 2006; 84: 2316-37.

2. McMillen IC, Robinson JS. Developmental origins of the metabolic syndrome: prediction, plasticity, and programming. Physiological reviews. 2005; 85: 571-633.

3. Aucott SW, Donohue PK, Northington FJ. Increased morbidity in severe early intrauterine growth restriction. Journal of perinatology. 2004; 24: 435-40.

4. Longo S, Bollani L, Decembrino L, Di Comite A, Angelini M, Stronati M. Short-term and long-term sequelae in intrauterine growth retardation (IUGR). The Journal of Maternal-Fetal \& Neonatal Medicine. 2013; 26: 222-5.

5. Hendrix N, Berghella V. Non-placental causes of intrauterine growth restriction. Seminars in perinatology: Elsevier; 2008. p. 161-5.

6. Raio L, Ghezzi F, Di Naro E, Duwe DG, Cromi A, Schneider H. Umbilical Cord Morphologic Characteristics and Umbilical Artery Doppler Parameters in Intrauterine Growth-Restricted Fetuses. Journal of ultrasound in medicine. 2003; 22: 1341-7.

7. Gayatri R, Crasta J, Thomas T, Pratibha D, Thomas A, Sridhar TS, et al. Structural Analysis of the Umbilical Cord and Its Vessels in Intrauterine Growth Restriction and Pre-eclampsia. Journal of Fetal Medicine. 2017: $1-8$.

8. Carmeliet P, Jain RK. Angiogenesis in cancer and other diseases. Nature. 2000; 407: 249.

9. Carmeliet $\mathrm{P}$, Jain RK. Principles and mechanisms of vessel normalization for cancer and other angiogenic diseases. Nature Reviews Drug Discovery. 2011; 10: 417-27.

10. Pegtel DM, Cosmopoulos K, Thorley-Lawson DA, van Eijndhoven MA, Hopmans ES, Lindenberg JL, et al. Functional delivery of viral miRNAs via exosomes. Proceedings of the National Academy of Sciences. 2010; 107: 6328-33.

11. Valadi H, Ekström $\mathrm{K}$, Bossios A, Sjöstrand M, Lee JJ, Lötvall JO. Exosome-mediated transfer of mRNAs and microRNAs is a novel mechanism of genetic exchange between cells. Nature cell biology. 2007; 9: 654-9.
12. Simons M, Raposo G. Exosomes-vesicular carriers for intercellular communication. Current opinion in cell biology. 2009; 21: 575-81.

13. Mathivanan S, Simpson RJ. ExoCarta: A compendium of exosomal proteins and RNA. Proteomics. 2009; 9: 4997-5000.

14. Li J, Zhang Y, Li D, Liu Y, Chu D, Jiang X, et al. Small non-coding RNAs transfer through mammalian placenta and directly regulate fetal gene expression. Protein \& cell. 2015; 6: 391-6.

15. Ouyang Y, Mouillet J-F, Coyne CB, Sadovsky Y. Review: placenta-specific microRNAs in exosomes-good things come in nano-packages. Placenta. 2014; 35: S69-S73.

16. Gallo A, Tandon M, Alevizos I, Illei GG. The majority of microRNAs detectable in serum and saliva is concentrated in exosomes. PloS one. 2012; 7: e30679.

17. Meng $X$, Lindsay $D$, Sriranganathan N. Wild boars as sources for infectious diseases in livestock and humans. Philosophical Transactions of the Royal Society of London B: Biological Sciences. 2009; 364: 2697-707.

18. Lunney JK. Advances in swine biomedical model genomics. Int J Biol Sci. 2007; 3: 179-84.

19. Andersson L. How selective sweeps in domestic animals provide new insight into biological mechanisms. Journal of internal medicine. 2012; 271: $1-14$

20. Che L, Thymann TS. IUGR does not predispose to necrotizing enterocolitis or compromise postnatal intestinal adaptation in preterm pigs. Pediatric Research. 2010; 67: 54-9.

21. Théry C, Amigorena S, Raposo G, Clayton A. Isolation and characterization of exosomes from cell culture supernatants and biological fluids. Current protocols in cell biology. 2006: 3.22. 1-3.. 9.

22. Palanisamy V, Sharma S, Deshpande A, Zhou H, Gimzewski J, Wong DT. Nanostructural and transcriptomic analyses of human saliva derived exosomes. PloS one. 2010; 5: e8577.

23. Li Y, Wang H-Y, Wan F-C, Liu F-J, Liu J, Zhang N, et al. Deep sequencing analysis of small non-coding RNAs reveals the diversity of microRNAs and piRNAs in the human epididymis. Gene. 2012; 497: 330-5.

24. Chen C-P, Bajoria R, Aplin JD. Decreased vascularization and cell proliferation in placentas of intrauterine growth-restricted fetuses with abnormal umbilical artery flow velocity waveforms. American journal of obstetrics and gynecology. 2002; 187: 764-9.

25. Che L, Thymann T, Bering SB, Huërou-Luron I LE, D'Inca R, Zhang K, et al. IUGR does not predispose to necrotizing enterocolitis or compromise postnatal intestinal adaptation in preterm pigs. Pediatric Research. 2010; 67: 54-9.

26. Sibley CP, Turner MA, Cetin I, Ayuk P, Boyd CA, Dsouza SW, et al. Placental phenotypes of intrauterine growth. Pediatric Research. 2005; 58: 827-32.

27. Regnault TRH, Galan HL, Parker TA, Anthony RV. Placental Development in Normal and Compromised Pregnancies - A Review. Placenta. 2002; 23: S119-S29.

28. Arroyo J, Winn V. Vasculogenesis and Angiogenesis in the IUGR Placenta. Seminars in Perinatology. 2008; 32: 172-7.

29. Torry DS, Hinrichs M, Torry RJ. Determinants of Placental Vascularity. American Journal of Reproductive Immunology. 2015; 51: 257-68.

30. Kosaka N, Izumi $H$, Sekine $K$, Ochiya T. microRNA as a new immune-regulatory agent in breast milk. Silence. 2010; 1: 1 .

31. Matsui J, Wakabayashi T, Asada M, Yoshimatsu K, Okada M. Stem cell factor/c-kit signaling promotes the survival, migration, and capillary tube formation of human umbilical vein endothelial cells. Journal of Biological Chemistry. 2004; 279: 18600-7.

32. Du T, Cheng J, Zhong L, Zhao XF, Zhu J, Zhu YJ, et al. The alleviation of acute and chronic kidney injury by human Wharton's jelly-derived mesenchymal stromal cells triggered by ischemia-reperfusion injury via an endocrine mechanism. Cytotherapy. 2012; 14: 1215-27.

33. Nargesi AA, Lerman LO, Eirin A. Mesenchymal stem cell-derived extracellular vesicles for renal repair. Current Gene Therapy. 2017; 17.

34. Zhang J, Li S, Li L, Li M, Guo C, Yao J, et al. Exosome and exosomal microRNA: trafficking, sorting, and function. Genomics, proteomics \& bioinformatics. 2015; 13: 17-24.

35. Berezikov E, Cuppen E, Plasterk RH. Approaches to microRNA discovery. Nature genetics. 2006; 38: S2-S7.

36. Chen Y, Gorski DH. Regulation of angiogenesis through a microRNA (miR-130a) that down-regulates antiangiogenic homeobox genes GAX and HOXA5. Blood. 2008; 111: 1217-26.

37. Wang S, Aurora AB, Johnson BA, Qi X, McAnally J, Hill JA, et al. The endothelial-specific microRNA miR-126 governs vascular integrity and angiogenesis. Developmental cell. 2008; 15: 261-71.

38. Reynolds LP, Borowicz PP, Vonnahme KA, Johnson ML, Grazul-Bilska AT, Redmer DA, et al. Placental angiogenesis in sheep models of compromised pregnancy. The Journal of physiology. 2005; 565: 43-58. 
39. Reynolds L, Ferrell C, Robertson DA, Ford S. Metabolism of the gravid uterus, foetus and utero-placenta at several stages of gestation in cows. The Journal of Agricultural Science. 1986; 106: 437-44.

40. Beck F. Vertebrate fetal membranes. Journal of anatomy. 1988; 156: 235.

41. Kosaka N, Iguchi H, Yoshioka Y, Takeshita F, Matsuki Y, Ochiya T. Secretory Mechanisms and Intercellular Transfer of MicroRNAs in Living Cells. Journal of Biological Chemistry. 2010; 285: 17442-52.

42. Zhang Y, Liu D, Chen X, Li J, Li L, Bian Z, et al. Secreted Monocytic miR-150 Enhances Targeted Endothelial Cell Migration. Molecular Cell. 2010; 39: 133-44. 